\title{
Erratum to: Uptake of a fluorescent L-glucose derivative 2-NBDLG into three-dimensionally accumulating insulinoma cells in a phloretin-sensitive manner
}

\author{
Ayako Sasaki $^{1}$ - Katsuhiro Nagatomo ${ }^{1} \cdot$ Koki Ono $^{1} \cdot$ Toshihiro Yamamoto $^{2,3}$. \\ Yuji Otsuka $^{2} \cdot$ Tadashi Teshima $^{2} \cdot$ Katsuya Yamada $^{1}$
}

Published online: 17 February 2016

(c) Japan Human Cell Society and Springer Japan 2016

\section{Erratum to: Human Cell (2016) 29:37-45 \\ DOI 10.1007/s13577-015-0125-3}

In the original publication of the article, Fig. 4k was published incorrectly. The correct figure is given in this erratum. The authors regret their errors.

The online version of the original article can be found under doi:10.1007/s13577-015-0125-3.

Katsuya Yamada

kyamada@hirosaki-u.ac.jp

1 Department of Physiology, Hirosaki University Graduate School of Medicine, 5 Zaifu-cho, Hirosaki,

Aomori 036-8562, Japan

2 Peptide Institute, Inc., Saito Research Center, Ibaraki, Osaka 567-0085, Japan

3 Graduate School of Science, Osaka University, Toyonaka, Osaka 560-0043, Japan 


\section{2 minutes after washout}

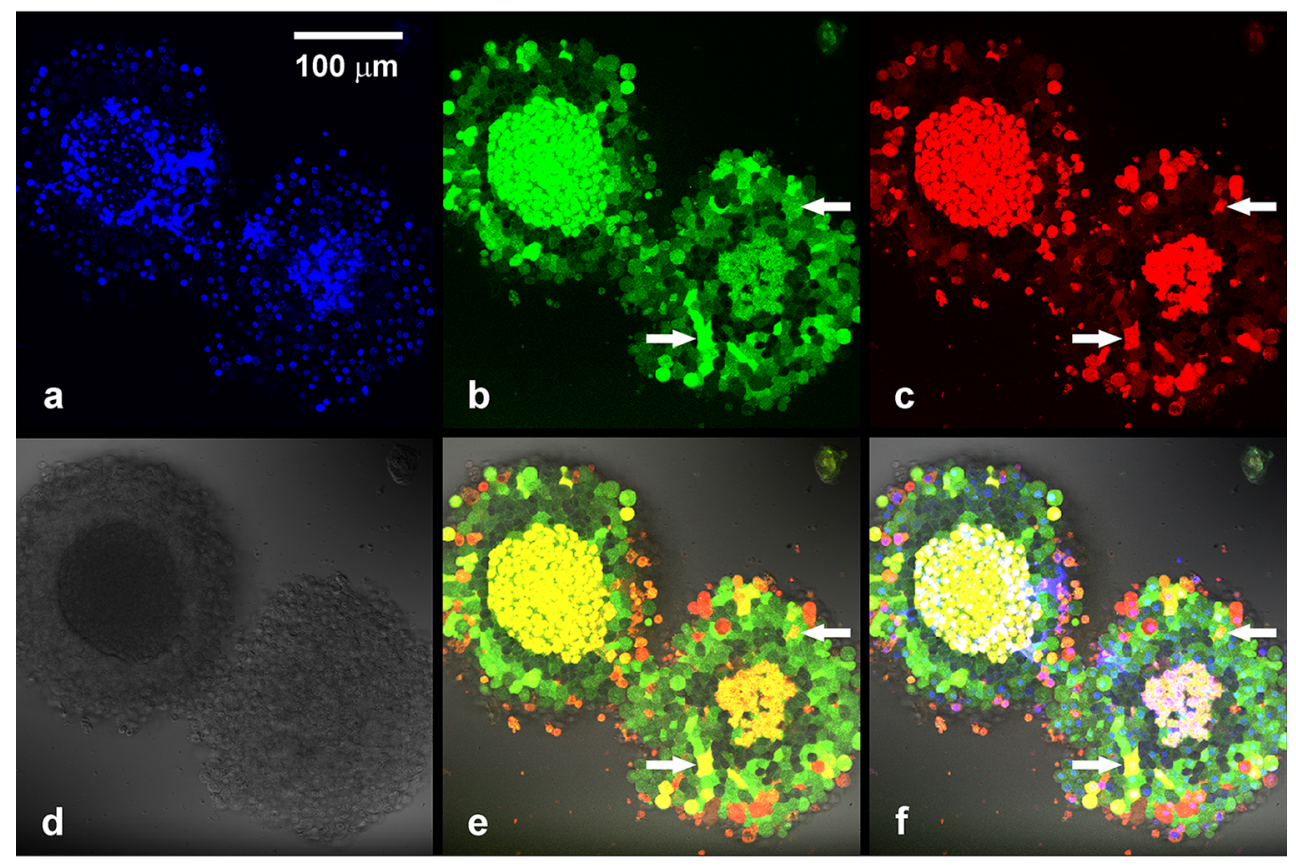

\section{4 minutes after washout}

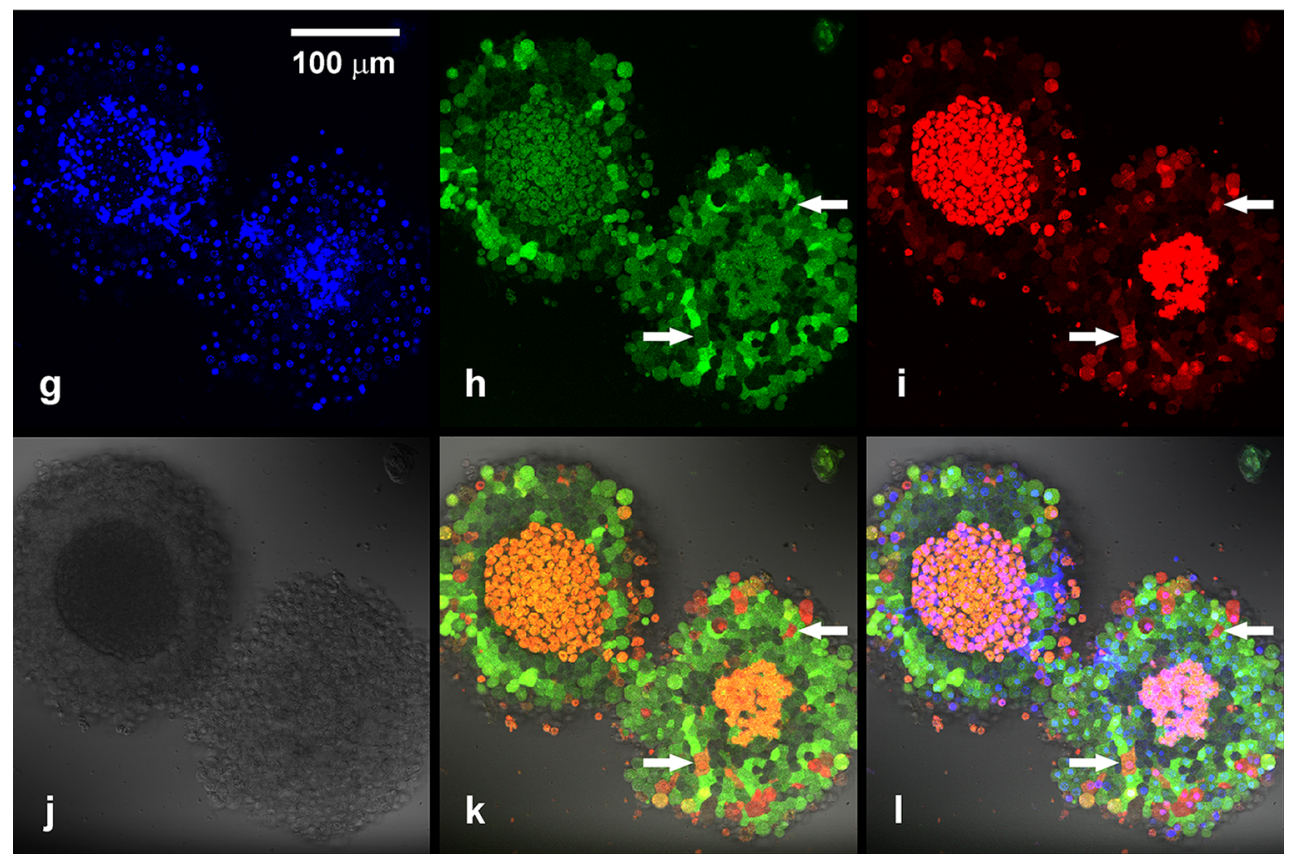

Fig. 4 Confocal microscopic images of 12 DIV MIN6 spheroids subjected to $100 \mu \mathrm{M}$ of 2-NBDLG (green) and $20 \mu \mathrm{M}$ of 2-TRLG (red) mixture for 3 min followed by washout. a Nuclear staining with DAPI in live cell condition. The central core region of spheroids appears to be necrotic (see also d). b and $\mathbf{c}$ Fluorescence images taken at $2 \mathrm{~min}$ after starting washout of the tracers in the green (b, $500-580 \mathrm{~nm}$ ) and the red (c, 580-740 nm) channel, reflecting entrance of 2-NBDLG and 2-TRLG, respectively. d Differential interference contrast (DIC) image. e Overlay of the green, red, and DIC images. f Overlay of (a) and (e). Cellular heterogeneity is clearly seen by a combination of the two fluorescent colors. Cells indicated by arrows exhibited yellow color at $2 \min (\mathbf{e})$, turned red at $4 \min (\mathbf{k})$. This is because green 2-NBDLG was lost $(\mathbf{b}, \mathbf{h})$, while red 2-TRLG remained $(\mathbf{c}, \mathbf{i})$. If one saw a single 2-NBDLG image $(\mathbf{b})$, cells indicated by arrows would have been misinterpreted to be similar to cells nearby. g- $\mathbf{I}$ Similar to $\mathbf{a}-\mathbf{f}$ but images taken at $4 \mathrm{~min}$ after starting washout. Numbers of green cells with no red fluorescence, seen in the area surrounding the central core, preserved their color for at least up to $30 \mathrm{~min}(\mathbf{h}, \mathbf{i}, \mathbf{k})$. Also noted are dark cells in the area just surrounding the central core $(\mathbf{b}, \mathbf{e}, \mathbf{h}, \mathbf{k})$. Bars are common to all panels 